\title{
Conhecimento e motivação de idosos sobre o atendimento geriátrico em serviço público de Teresina/PI
}

\author{
Knowledge and motivation of the elderly about geriatric care in public service in Teresina/PI \\ Conocimiento y motivación del mayor sobre el cuidado geriátrico en el servicio público en
}

Teresina/PI

Recebido: 06/12/2021 | Revisado: 18/12/2021 | Aceito: 19/12/2021 | Publicado: 02/01/2022

\author{
Rawenna Machado Dias De Oliveira \\ ORCID: https://orcid.org/0000-0002-3175-8717 \\ Centro Universitário Uninovafapi, Brasil \\ E-mail: rawenna_123@hotmail.com \\ João José Moura Barros \\ ORCID: https://orcid.org/0000-0002-9984-7177 \\ Centro Universitário Uninovafapi, Brasil \\ E-mail: joaojosembarros@outlook.com \\ João Luiz Vieira Ribeiro \\ ORCID: https://orcid.org/0000-0003-4243-810X \\ Centro Universitário Uninovafapi, Brasil \\ E-mail: jlvribeiro@yahoo.com
}

\begin{abstract}
Resumo
Objetivo: Analisar o conhecimento de pessoas com idade igual ou superior a 60 anos sobre a especialidade geriatria e a motivação destes para a procura de atendimento especializado com o profissional geriatra. Metodologia: Este é um estudo qualitativo, quantitativo, descritivo, analítico e transversal. Amostra: 357 idosos, grau de confiança: $95 \%$ $(\mathrm{z}=1,96), \mathrm{E}=5 \%, \mathrm{p}=0,23 \mathrm{e} \mathrm{N}=5000$. Como instrumento de pesquisa foi utilizado um questionário. Resultados: $55,5 \%$ concordam totalmente sobre a existência de uma especialidade para cuidar do envelhecimento, 75,4\% discordam totalmente sobre a recomendação de um profissional à consulta com geriatra, $15,4 \%$ se consultou com este profissional, 40,6\% têm alguma limitação para se consultar com um geriatra, incluindo transporte, distância e renda como os fatores mais mencionados pelos entrevistados. Conclusão: Para os idosos incluídos neste estudo, existem obstáculos tanto no conhecimento a respeito da geriatria e quanto na motivação para estes idosos realizarem consultas com o profissional geriatra.
\end{abstract}

Palavras-chave: Geriatria; Motivação; Conhecimento; Consulta médica.

\begin{abstract}
Objective: To analyze the knowledge of people aged 60 years or over about the geriatric specialty and their motivation to seek specialized care from a geriatrician. Methodology: This is a qualitative, quantitative, descriptive, analytical and cross-sectional study. Sample: 357 elderly, confidence level: $95 \%(z=1.96), E=5 \%, p=0.23$ and $N=5000$. A questionnaire was used as a research instrument. Results: $55.5 \%$ totally agreed about the existence of a specialty to care for aging, $75.4 \%$ totally disagreed about the recommendation of a professional to see a geriatrician, $15.4 \%$ had a consultation with this professional, $40.6 \%$ some limitation in seeing a geriatrician, including transport, distance and income as the factors most identified by respondents. Conclusion: For the elderly included in this study, there is as much knowledge in knowledge about geriatrics as there is motivation for these elderly people to consult a geriatrician. Keywords: Geriatrics; Motivation; Knowledge; Medical appointment.

\section{Resumen}

Objetivo: Analizar el conocimiento de personas de 60 años o más sobre la especialidad geriátrica y su motivación para buscar atención especializada de un geriatra. Metodología: Se trata de un estudio cualitativo, cuantitativo, descriptivo, analítico y transversal. Muestra: 357 ancianos, nivel de confianza: 95\% $(\mathrm{z}=1,96), \mathrm{E}=5 \%, \mathrm{p}=0,23$ y $\mathrm{N}=5000$. Se utilizó un cuestionario como instrumento de investigación. Resultados: El 55,5\% estuvo totalmente de acuerdo con la existencia de una especialidad para el cuidado del envejecimiento, el 75,4\% estuvo totalmente en desacuerdo con la recomendación de un profesional para ver a un geriatra, el 15,4\% tuvo una consulta con este profesional, el 40,6\% alguna limitación para ver a un geriatra, incluyendo el transporte, la distancia y los ingresos como los factores más identificados por los encuestados. Conclusión: Para las personas mayores incluidas en este estudio, existe tanto conocimiento en el conocimiento sobre geriatría como motivación para que estas personas mayores consulten a un geriatra.
\end{abstract}

Palavras clave: Geriatría; Motivación; Conocimiento; Consulta médica. 


\section{Introdução}

Idoso é todo aquele que apresenta 60 anos ou mais, conforme definição da Organização Mundial de Saúde - OMS. (Machado, 2019) Esta população, a partir de 2018 se tornou maior que a população de crianças com idade inferior a cinco anos de idade, segundo a Organização das Nações Unidas. (ONU) As projeções indicam a possibilidade de que, em 2050, o número de idosos seja duas vezes maior que o de crianças com idade igual ou menor que 5 anos de idade e há a chance de que ultrapasse, também, o número de adolescentes e jovens de 15 a 24 anos. (United Nations, 2019) No Brasil, tal transição demográfica se dá, dentre outras causas, pelo envelhecimento progressivo de sua população devido ao aumento da expectativa de vida. (UNFPA,2018)

A longevidade traz consigo desafios e oportunidades, sendo todos estes, diretamente ou indiretamente dependentes da saúde. Por isso, é de grande importância que os serviços de saúde estejam de acordo, também, com as necessidades inerentes ao grupo de pessoas idosas. (OPAS/OMS Brasil, 2021) Desse modo, profissionais geriatras e gerontologistas tornam-se importantes protagonistas na promoção de saúde, visto que estes são responsáveis pelos cuidados de saúde com esta parcela da população, desde a promoção, manutenção, tratamento e reabilitação das doenças específicas do envelhecimento saudável. (SBGG, 2021)

Desde a Constituição Federal de 1988, conhecida como “Constituição Cidadã”, os idosos ganham normas protetivas aos seus direitos como saúde e integridade. A partir dela, surgiram várias políticas específicas para os idosos, a exemplo, a Política Nacional do Idoso (1994), a Política Nacional de Saúde do Idoso (1999) e o Estatuto do Idoso (2003). Tais políticas são de grande importância para a promoção da qualidade de vida e melhora da capacidade funcional desses idosos. (Veras \& Oliveira, 2018)

Dentre elas, é possível destacar a importância da Política Nacional do Idoso (Lei Federal 8.842, de 1994) que objetiva assegurar, dentre outros direitos, esse acesso à saúde pela população com idade igual ou superior a 60 anos. Para isso, esta defende a inclusão da Geriatria e da Gerontologia nos recursos humanos e na prestação de serviços, em concursos e como disciplina de cursos superiores. Portanto, essa política foi de grande importância para a formação em Geriatria e Gerontologia para que, a partir de então, existam profissionais qualificados voltados para o atendimento a esta população. (Brasil, 1994)

Mesmo com o surgimento de políticas públicas que promovam a qualidade de vida entre os idosos, diversos fatores individuais acumulados ao longo da vida mostram desigualdades que são verdadeiros desafios à promoção da saúde. Estes estão relacionados a fatores físicos, ambientais e sociais de cada um desses indivíduos. Sendo assim, é de grande importância que os sistemas de saúde estejam prontos para fortalecer os cuidados centrados na pessoa idosa. (OPAS/OMS Brasil, 2021)

Conforme dados do último Censo do IBGE - Instituto Brasileiro de Geografia e Estatística - (2010), a população do Brasil, é igual a 190.755.799 pessoas. Destas, 20.588 .891 possuem 60 anos ou mais, o que equivale a aproximadamente $10.80 \%$ da população brasileira. (IBGE, 2010) Para o atendimento geriátrico dessas pessoas, segundo o Conselho Regional de Medicina (CRM), possuem 1.855 geriatras em situação regular no Brasil. (CRM-PI, 2020) Por meio de um cálculo simples, percebe-se que existem apenas 0.0000900971 geriatras em todo o país para cada idoso. Esses dados reforçam a importância desse profissional como meio para fortalecimento do sistema de saúde do País.

Paralelamente, segundo o IBGE (2010), a população de Teresina, capital do Piauí, é de 814.230 pessoas, sendo que, destes, temos o equivalente a 69.122 pessoas com idade igual ou superior a 60 anos, o que equivale a aproximadamente $8,49 \%$ da população local. (IBGE, 2010) Assim, percebe-se a necessidade de cuidado integrado em saúde para esta população teresinense com 60 anos ou mais.

Conforme o Conselho Regional de Medicina do Estado do Piauí (CRM-PI), em Teresina encontram-se 19 registros de profissionais médicos geriatras, que, após uma simples divisão, resultam em apenas 0.27487 geriatras para todos os idosos 
teresinenses. Para ficar mais claro, este número mostra que, se cada idoso que mora em Teresina fosse a 1 geriatra, cada geriatra atenderia o equivalente a 3.638 idosos. (CRM-PI, 2020)

Visto a importância do geriatra na vida dessa população, este trabalho de conclusão de curso tem como objetivo avaliar o conhecimento de idosos a respeito da existência da especialidade médica Geriatria e a motivação desse público para procurar atendimento com o profissional geriatra em serviço público de Teresina.

\section{Metodologia}

O estudo possui uma abordagem qualitativa e quantitativa do tipo descritivo, analítico e transversal de levantamento de dados, relativos ao nível de conhecimento de idosos sobre o serviço de geriatria e a motivação para buscar o atendimento com o profissional geriatra. (Pereira et al., 2018) Utilizou-se como único instrumento de coleta de dados um questionário anônimo.

A população do estudo envolve 5000 idosos que frequentam o Centro Integrado de Saúde (CIS), do Centro Universitário Uninovafapi em Teresina/Piauí. Desses foi selecionada a amostra de 357 pacientes idosos, aleatoriamente, enquanto aguardavam atendimento nos turnos matutino e vespertino, realizado no período de 01 de junho a 30 de setembro de 2021. Para determinar este número foi usada a fórmula: $\mathrm{n}=\left(\mathrm{z}^{2} \cdot \mathrm{p} \cdot(1-\mathrm{p}) \cdot \mathrm{N}\right) /\left(\mathrm{E}^{2}(\mathrm{~N}-1)+\mathrm{z}^{2} \cdot \mathrm{p} \cdot(1-\mathrm{p})\right)=$ $\left.\left(1,96^{2} \cdot 0,23 \cdot 0,77 \cdot 5000\right) /\left(0,05^{2} \cdot 4999+1,96^{2} \cdot 0,23 \cdot 0,77\right)\right)$, na qual, z é o valor crítico, E a margem de erro e $\mathrm{N}$ o tamanho da população, considerando o grau de confiança de $95 \%(z=1,96)$, margem de erro $E=5 \%$, proporção $p=0,23$ e N = 5000.

Os critérios de inclusão adotados foram os participantes possuírem idade igual ou superior a sessenta anos e serem capazes de assinar o nome, ou, em caso de impossibilidade de assinar o TCLE, foram aceitos ou a impressão digital do participante na linha de assinatura ou a assinatura do responsável do participante. Como critérios de exclusão adotou-se a presença de deficiências significativas como o comprometimento das funções cognitivas ou auditivas e dificuldade de compreensão.

Os questionários foram apresentados pelos autores aos participantes. Em seguida, foram lidos para os pacientes, explicado quando necessário e os próprios pesquisadores anotaram as respostas. Primeiramente, foram inqueridos quanto ao gênero, faixa etária, estado civil, escolaridade e região onde mora em questões de resposta única. Posteriormente foram submetidos a outras 12 afirmações para avaliação acerca do conhecimento da existência do serviço de geriatria e a motivação para busca de um geriatra, sendo 10 respondidas conforme a escala de Likert de concordância, 2 questões dicotômicas e 1 aberta. A duração do preenchimento dos questionários foi de aproximadamente 15 minutos.

Dados sociodemográficos, da secção 1 do formulário, foram analisados por meio de estatísticas descritivas, através das médias, mediana e desvio padrão. Os resultados do formulário aplicado da secção 2 foram registrados na planilha Microsoft Excel, que posteriormente foi exportada para o programa IBM SPSS que fez o processamento dos dados estatísticos e fornecerá resultados em gráficos e tabelas por meio de frequências absolutas $(\mathrm{N})$ e frequências relativas (\%).

Todos os idosos participantes da amostra assinaram o Termo de Consentimento Livre e Esclarecido, ou, em caso de impossibilidade de qualquer espécie, foi aceito a impressão digital na linha de assinatura ou a assinatura do responsável pelo participante. Também foi comunicado sobre o tema da pesquisa assim como esclarecidos da escolha de participar. A pesquisa foi iniciada apenas após a aprovação do Comitê de Ética em Pesquisa do Centro Universitário Uninovafapi e submissão a Plataforma Brasil. Dessa forma, foram cumpridos todos os preceitos éticos da Resolução no 196 do Conselho Nacional de Saúde de 10 de outubro de 1996.

Para participar deste estudo o participante não teve custos e não recebeu vantagens financeiras. Esta pesquisa não envolveu quaisquer riscos significativos, além do risco de quebra de anonimato. Para prevenir e minimizar este risco, o questionário apresentado teve caráter anônimo, sendo numerado apenas para organização. Os benefícios obtidos por meio deste 
estudo são indiretos, pois as informações coletadas ajudarão a delinear novas pesquisas em Medicina. Estas informações serão usadas apenas para fins científicos.

\section{Resultados}

Foram entrevistados 357 idosos que frequentam o Centro Integrado de Saúde (CIS), do Centro Universitário Uninovafapi em Teresina/Piauí. A respeito da caracterização socioeconômica dos entrevistados (Tabela 1), 206 eram do gênero feminino, o que corresponde ao valor de $57,7 \%$ do total de entrevistados. Os demais $42,3 \%$ se autodeclararam do gênero masculino. 68,9\% dos entrevistados possuíam idades entre 60 e 70 anos, seguidos por $24,08 \%$ com idades entre 71 e 80 anos e 25 com idades iguais ou superiores a 81 anos. 54,9\% dos entrevistados eram casados, seguidos por 18,3\% viúvos, 14,3\% solteiros e $9,8 \%$ divorciados. Os outros $2,8 \%$ declararam seu estado civil como "OUTROS". No que diz respeito a escolaridade, 157 participantes da entrevista $(44,0 \%)$ possuíam $1^{\circ}$ grau incompleto, seguido de $19,9 \%$ com $2^{\circ}$ grau completo e outros $14,0 \%$ não alfabetizados. Quanto aos demais, 10,6\% possuíam $1^{\circ}$ grau completo, $6,4 \%$ com terceiro grau completo, $4,2 \% \operatorname{com} 2^{\circ}$ grau incompleto e $0,8 \%$ com $3^{\circ}$ grau incompleto. 167 entrevistados residiam na região leste de Teresina (46,8\%), seguidos de pessoas que moravam em outras regiões $21 \%$ (tais como zona rurais, outros municípios do estado e/ou do Nordeste). A respeito dos pacientes que residiam nas zonas sudeste, sul, norte e centro, tivemos, respectivamente: $12,9 \%$; $11,5 \% 5,6 \%$ e $2,2 \%$ dos entrevistados.

Tabela 1 - Perfil sociodemográfico dos idosos que frequentam o Centro Integrado de (CIS), do Centro Universitário Uninovafapi em Teresina/Piauí.

\begin{tabular}{|c|c|c|c|}
\hline Variável & & Frequência & $\%$ \\
\hline \multirow{2}{*}{ Gênero } & FEMININO & 206 & $57,7 \%$ \\
\hline & MASCULINO & 151 & $42,3 \%$ \\
\hline \multirow{3}{*}{ Idade } & 60-70 ANOS & 246 & $68,9 \%$ \\
\hline & 71-80 ANOS & 85 & $23,8 \%$ \\
\hline & $>80$ ANOS & 25 & $7,0 \%$ \\
\hline \multirow{5}{*}{ Estado Civil } & CASADO & 196 & $54,9 \%$ \\
\hline & DIVORCIADO & 35 & $9,8 \%$ \\
\hline & OUTROS & 10 & $2,8 \%$ \\
\hline & SOLTEIRO & 51 & $14,3 \%$ \\
\hline & VIUVO & 65 & $18,2 \%$ \\
\hline \multirow{7}{*}{ Escolaridade } & $1^{\circ}$ GRAU COMPLETO & 38 & $10,6 \%$ \\
\hline & $1^{\circ}$ GRAU INCOMPLETO & 157 & $44,0 \%$ \\
\hline & $2^{\circ}$ GRAU COMPLETO & 71 & $19,9 \%$ \\
\hline & $2^{\circ}$ GRAU INCOMPLETO & 15 & $4,2 \%$ \\
\hline & $3^{\circ}$ GRAU COMPLETO & 23 & $6,4 \%$ \\
\hline & $3^{\circ}$ GRAU INCOMPLETO & 3 & $0,8 \%$ \\
\hline & NÃO ALFABETIZADO & 50 & $14,0 \%$ \\
\hline \multirow{6}{*}{ Região em que mora } & CENTRO & 8 & $2,2 \%$ \\
\hline & LESTE & 167 & $46,8 \%$ \\
\hline & NORTE & 20 & $5,6 \%$ \\
\hline & OUTRO & 75 & $21,0 \%$ \\
\hline & SUDESTE & 46 & $12,9 \%$ \\
\hline & SUL & 41 & $11,5 \%$ \\
\hline
\end{tabular}

Fonte: Autores.

A respeito dos questionários que abordava o conhecimento a respeito da geriatria como especialidade médica e a motivação desses idosos para frequentar à consulta, tivemos os seguintes resultados (Tabela 2): 
1. $198(55,5 \%)$ entrevistados concordaram totalmente a respeito da existência de uma especialidade médica para cuidar do envelhecimento. Este número foi seguido por $24,1 \%$ dos pacientes que não estavam decididos a respeito da existência de uma especialidade para cuidar do envelhecimento.

2. $175(49,05)$ entrevistados afirmam concordar totalmente que o geriatra/gerontologista é o especialista que cuida do envelhecimento. Este número é seguido por 129 (36,1\%) dos entrevistadoss que afirmam não estar decididos a respeito desta afirmação.

3. 162 entrevistados $(45,4 \%)$ concordam totalmente que "O médico geriatra/gerontologista é o médico que cuida desde a promoção, manutenção, tratamento e reabilitação das doenças específicas do envelhecimento", enquanto outros 135 $(37,8 \%)$ afirma não estar decidido a respeito desta afirmação.

4. 269 pacientes $(75,4 \%)$ discordam totalmente quando interrogados se algum profissional de saúde já o recomendou uma consulta com o médico geriatra, contra 20,2\% que concordam totalmente a respeito do mesmo questionamento.

5. $284(79,8 \%)$ dos entrevistados concordam totalmente que consideram importante ir ao médico geriatra, outros 15 pacientes discordam (totalmente $3,4 \%$ e parcialmente $0,8 \%$ ) a respeito da importância da consulta com este médico. Outros 28 pacientes não estão decididos a respeito da importância desta consulta $(7,9 \%)$.

6. O total de 302 entrevistados afirmam nunca terem se consultado com médico geriatra. Do total de entrevistados, $15,4 \%$ já se consultou em algum momento com este profissional.

7. $314(88,0 \%)$ entrevistados discordam totalmente a respeito da regularidade das consultas com o médico geriatra ao longo de um ano, este número é seguido por 30 pacientes que afirmam ter regularidade em suas consultas anuais, sendo que 6 $(1,7 \%)$ entrevistados concordam parcialmente e outros $24(6,7 \%)$, totalmente.

8. 212 entrevistados discordam totalmente a respeito da afirmação "Vou ao médico geriatra apenas quando encaminhado por outro especialista médico", outros 72 entrevistados (20,2\%) afirmam concordar totalmente com a afirmação.

9. 213 pacientes concordam totalmente na crença de que nunca precisaram se consultar com um médico geriatra $(59,7 \%)$. Outros $93(26,1 \%)$ pacientes discordam totalmente. Mais 24 entrevistados afirmam não estar decididos sobre a necessidade de consulta com o médico geriatra.

10. 237 entrevistados discordam totalmente sobre terem ido ao geriatra e terem ficado satisfeitos com o atendimento. 51 entrevistados concordam (0,3\% parcialmente e $14 \%$ totalmente) já terem ido ao geriatra e ficado satisfeitos com o atendimento.

11. A respeito da limitação para consulta com médico geriatra, $212(59,4 \%)$ afirmam não ter nenhuma limitação para a realização de uma consulta com o médico geriatra, contra 145 entrevistados (40,6\%) que afirmam ter alguma limitação para se consultar com o médico geriatra.

12. Foi deixado em aberto os fatores limitantes para aqueles pacientes que possuíam alguma limitação (40,6\%), sendo que podiam citar os que julgassem necessário, sem limite de quantidade de fatores. Dentre eles:

a. O termo "transporte" foi citado 28 vezes, somado a "ônibus" que foi citado 5 vezes, associado a "poucos", "greve", “ausência". O termo "transporte por aplicativo" foi citado associado a "alto valor" 1 vez

b. "SUS" foi citado sendo correlacionado a "demora para marcar consulta" 14 vezes, "espera para marcar consulta" 6 vezes, “dependência do SUS” 5 vezes, “dificuldade/ não conseguir marcar consulta pelo SUS” 6 vezes, "fila” 2 vezes, "alta demanda" 1 vez, "burocracia" 2 vezes, "precariedade do atendimento" 1 vez

c. O termo "acompanhante" foi citado 14 vezes

d. "Dificuldade/limitação financeira/ falta recursos/ dinheiro" foi citado 10 vezes

e. "Pandemia" foi citado 3 vezes, estando relacionada a "serviços parados" 1 vez, "medo de covid-19" 1 vez

f. "Distância" foi citado 21 vezes 
g. "Não conseguir marcar por convênios" 1 vez

h. "Não tem no bairro/ na região/na cidade" 5 vezes

i. "Novo na região em que mora" 1 vez

j. "Moro no interior/zona rural/outra cidade" 12 vezes

k. "Gastos associados a viagem para consultas" 1 vez

1. "Cansaço físico" $1 \mathrm{vez}$

m. "Desrespeito a idosos" $1 \mathrm{vez}$

n. "Não conheço onde tem profisssional" 3 vezes

o. "Pouco número de geriatra" 2 vezes

p. "Não precisa" foi citado 1 vez, associado a "se considera jovem/não se considera idoso" 5 vezes, "não sabe se precisa

q. Limitações de saúde: "deficiência física" 2 vezes, "artrose nas pernas/problemas articulares" 2 vezes, "doença da tireoide" 1 vez, "refluxo" 1 vez, "problema nas pernas" 2 vezes, "dificuldade para caminhar/se locomover" 3 vezes, “esquecimento" 1 vez, "problema de visão" 2 vezes, "dificuldade com audição" 3 vezes, "problema cardíaco" 1 vez, “dificuldade para viajar/passa mal em viagens" 1 vez, "cadeirante" 3 vezes, "pés dormentes" 1 vez

r. "Não tenho conhecimento a especialidade" 10 vezes

s. "Não gosto de ir ao médico/ não gosta de consultas/ sente ansiedade ao ir a consultas" 5 vezes

t. "Não tenho plano de saúde/convênio" 5 vezes

u. "Valor da consulta" 3 vezes

v. "Analfabetismo/ dificuldade relacionada a alfabetização" 3 vezes

w. "Tempo" 2 vezes e "tempo do acompanhante" 2 vezes

x. "Realizo consultas com outras especialidades" 3 vezes e "Prefiro outro médico" 1 vez

Tabela 2 - Avaliação do conhecimento sobre a geriatria e motivação para buscar atendimento.

\begin{tabular}{|c|c|c|c|}
\hline Variável & & Frequência & $\%$ \\
\hline \multirow{5}{*}{$\begin{array}{l}\text { Existe uma especialidade médica para cuidar do } \\
\text { envelhecimento }\end{array}$} & CONCORDO PARCIALMENTE & 31 & $8,7 \%$ \\
\hline & CONCORDO TOTALMENTE & 198 & $55,5 \%$ \\
\hline & DISCORDO PARCIALMENTE & 8 & $2,2 \%$ \\
\hline & DISCORDO TOTALMENTE & 34 & $9,5 \%$ \\
\hline & NÃO ESTOU DECIDIDO & 86 & $24,1 \%$ \\
\hline \multirow{5}{*}{$\begin{array}{l}\text { O geriatra/gerontologista é o especialista que cuida } \\
\text { do envelhecimento }\end{array}$} & CONCORDO PARCIALMENTE & 33 & $9,2 \%$ \\
\hline & CONCORDO TOTALMENTE & 175 & $49,0 \%$ \\
\hline & DISCORDO PARCIALMENTE & 7 & $2,0 \%$ \\
\hline & DISCORDO TOTALMENTE & 13 & $3,6 \%$ \\
\hline & NÃO ESTOU DECIDIDO & 129 & $36,1 \%$ \\
\hline \multirow{7}{*}{$\begin{array}{l}\text { O médico geriatra/gerontologista é o médico que } \\
\text { cuida desde a promorção, manutenção, tratamento e } \\
\text { reabilitação das doenças específicas do } \\
\text { envelhecimento. }\end{array}$} & CONCORDO PARCIALMENTE & 41 & $11,5 \%$ \\
\hline & CONCORDO TOTALMENTE & 162 & $45,4 \%$ \\
\hline & DISCORDO PARCIALMENTE & 5 & $1,4 \%$ \\
\hline & DISCORDO TOTALMENTE & 14 & $3,9 \%$ \\
\hline & NÃO ESTOU DECIDIDO & 135 & $37,8 \%$ \\
\hline & DISCORDO TOTALMENTE & 269 & $75,4 \%$ \\
\hline & NÃO ESTOU DECIDIDO & 14 & $3,9 \%$ \\
\hline \multirow{4}{*}{$\begin{array}{l}\text { Algum profissional de saúde já me recomendou a } \\
\text { consulta com o médico geriatra }\end{array}$} & CONCORDO PARCIALMENTE & 2 & $0,6 \%$ \\
\hline & CONCORDO TOTALMENTE & 72 & $20,2 \%$ \\
\hline & DISCORDO TOTALMENTE & 269 & $75,4 \%$ \\
\hline & NÃO ESTOU DECIDIDO & 14 & $3,9 \%$ \\
\hline \multirow{5}{*}{ Considero importante ir ao médico geriatra } & CONCORDO PARCIALMENTE & 30 & $8,4 \%$ \\
\hline & CONCORDO TOTALMENTE & 284 & $79,6 \%$ \\
\hline & DISCORDO PARCIALMENTE & 3 & $0,8 \%$ \\
\hline & DISCORDO TOTALMENTE & 12 & $3,4 \%$ \\
\hline & NÃO ESTOU DECIDIDO & 28 & $7,8 \%$ \\
\hline
\end{tabular}




\begin{tabular}{|c|c|c|c|}
\hline Já me consultei com médico geriatra & $\begin{array}{c}\text { NÃO } \\
\text { SIM }\end{array}$ & $\begin{array}{c}302 \\
55\end{array}$ & $\begin{array}{l}84,6 \% \\
15,4 \% \\
\end{array}$ \\
\hline $\begin{array}{l}\text { Vou regularmente ao médico geriatra ao longo de } \\
\text { um ano }\end{array}$ & $\begin{array}{c}\text { CONCORDO PARCIALMENTE } \\
\text { CONCORDO TOTALMENTE } \\
\text { DISCORDO PARCIALMENTE } \\
\text { DISCORDO TOTALMENTE } \\
\text { NÃO ESTOU DECIDIDO }\end{array}$ & $\begin{array}{c}6 \\
24 \\
11 \\
314 \\
2 \\
\end{array}$ & $\begin{array}{c}1,7 \% \\
6,7 \% \\
3,1 \% \\
88,0 \% \\
0,6 \% \\
\end{array}$ \\
\hline $\begin{array}{l}\text { Vou ao médico geriatra apenas quando encaminho } \\
\text { por outro especialista médico }\end{array}$ & $\begin{array}{c}\text { CONCORDO PARCIALMENTE } \\
\text { CONCORDO TOTALMENTE } \\
\text { DISCORDO PARCIALMENTE } \\
\text { DISCORDO TOTALMENTE } \\
\text { NÃO ESTOU DECIDIDO } \\
\end{array}$ & $\begin{array}{c}8 \\
72 \\
24 \\
212 \\
41 \\
\end{array}$ & $\begin{array}{c}2,2 \% \\
20,2 \% \\
6,7 \% \\
59,4 \% \\
11,5 \% \\
\end{array}$ \\
\hline $\begin{array}{l}\text { Nunca precisei me consultar com um médico } \\
\text { geriatra }\end{array}$ & $\begin{array}{c}\text { CONCORDO PARCIALMENTE } \\
\text { CONCORDO TOTALMENTE } \\
\text { DISCORDO PARCIALMENTE } \\
\text { DISCORDO TOTALMENTE } \\
\text { NÃO ESTOU DECIDIDO }\end{array}$ & $\begin{array}{c}12 \\
213 \\
15 \\
93 \\
24 \\
\end{array}$ & $\begin{array}{c}3,4 \% \\
59,7 \% \\
4,2 \% \\
26,1 \% \\
6,7 \% \\
\end{array}$ \\
\hline $\begin{array}{l}\text { Já fui ao geriatra e fiquei satisfeito com o } \\
\text { atendimento }\end{array}$ & $\begin{array}{c}\text { CONCORDO PARCIALMENTE } \\
\text { CONCORDO TOTALMENTE } \\
\text { DISCORDO PARCIALMENTE } \\
\text { DISCORDO TOTALMENTE } \\
\text { NÃO ESTOU DECIDIDO } \\
\end{array}$ & $\begin{array}{c}1 \\
50 \\
61 \\
237 \\
8 \\
\end{array}$ & $\begin{array}{c}0,3 \% \\
14,0 \% \\
17,1 \% \\
66,4 \% \\
2,2 \% \\
\end{array}$ \\
\hline $\begin{array}{l}\text { Tenho alguma limitação para me consultar com o } \\
\text { médico geriatra }\end{array}$ & $\begin{array}{c}\text { NÃO } \\
\text { SIM }\end{array}$ & $\begin{array}{l}212 \\
145\end{array}$ & $\begin{array}{l}59,4 \% \\
40,6 \%\end{array}$ \\
\hline
\end{tabular}

Fonte: Autores.

\section{Discussão}

Segundo dados da ONU, a população com 60 anos ou mais está cada vez maior no Brasil e no mundo e deve aumentar a mais que dobrar até o ano de 2050. Inerentes a esta mudança demográfica, estão os declínios na capacidade física e mental devido, principalmente, às doenças crônicas deste grupo populacional. (Sá et al., 2019)

Esse mesmo fenômeno é presenciado na capital do estado do Piauí, segundo dados do IBGE. Em 1991, a população teresinense era de 599.272 e a da população com mais de 60 anos era de 29.584, o que equivaleria a aproximadamente 4,93\% de toda a população. Em seguida, em 2010 (último censo), esta mesma população era de 69.122 idosos para o total de 814.230 pessoas, o que equivalia a aproximadamente $8,49 \%$ de toda a população teresinense. Sendo assim, percebe-se que em 19 anos Teresina aumentou 2,33\% sua taxa percentual de idosos. (IBGE, 2010)

Estes dados são importantes para esta pesquisa, visto que a Geriatria é a especialidade médica que cuida especificamente do grupo de pessoas pertencentes à faixa etária de 60 anos ou mais. Em Teresina, de acordo com os dados apresentados na página online do CRM-PI, tem-se o total de 19 geriatras. Sendo assim, podemos rapidamente, por meio de uma regra de três simples, demonstrar que se cada um desses geriatras fossem atender aos idosos de Teresina, eles teriam um total de 3.638 pacientes teresinenses.

Este último cenário (cada idoso teresinense realizando consultas geriátricas) seria o ideal, visto que o acesso à saúde é um direito de todos, conforme o Artigo 196 da Constituição Federal de 1988: “A saúde é direito de todos e dever do Estado, garantido mediante políticas sociais e econômicas que visem à redução do risco de doença e de outros agravos e ao acesso universal e igualitário às ações e serviços para sua promoção, proteção e recuperação”. (Brasil, 1988)

No entanto, a disponibilidade do médico geriatra, o acesso a ele e a disseminação do conhecimento acerca da existência desse profissional é de grande importância para que se cumpra não somente o direito estabelecido na Carta Magna, mas também para que se cumpra o princípio de equidade do SUS - Sistema Único de Saúde. (Araújo et al., 2017)

Para a faixa etária geriátrica, a equidade na saúde passa a ter realização por meio do acesso ao médico geriatra, pois será este o profissional que irá prover o atendimento específico e necessário para esta faixa etária. O Estatuto do Idoso, Lei 
$\mathrm{n}^{\circ} 10.741$, de $1^{\circ}$ de outubro de 2003, também reforça a importância, especificamente, do acesso ao médico geriatra como parte importante para assegurar a atenção integrada ao idoso por meio SUS. (Brasil, 2003)

Isto é de grande relevância visto que idosos tem particularidades fisiológicas que os diferem das demais faixas etárias sofrendo, portanto, por diagnósticos de difícil reconhecimento ou tratamentos inadequados quando atendidos por médicos que não tenham formação em saúde do idoso. (Heflin, 2020) Ademais, cada idoso deve ser visto como um ser único visto que o envelhecimento é um processo heterogêneo, multifatorial e que traz consigo modificações fisiológicas, funcionais, emocionais e, diversas vezes, também sociais e econômicas individuais. (Fonseca et al., 2016)

Outrossim, o geriatra é o médico especialista, que foi treinado e preparado para o atendimento de idosos. A importância deste médico está, também, no conhecimento da farmacologia a ser aplicada, visto que os fármacos atuam de forma particular nessa faixa etária. Assim, é necessário profundo conhecimento ao indicar ou não cada droga para cada idoso, de acordo com cada realidade. (Rochon, 2020)

Segundo este raciocínio, uma boa indicação de tratamento e/ou de medicamentos é de grande importância para a prevenção de eventos adversos evitáveis. Estes, estão relacionamentos a maior sobrecarga de trabalho de profissionais da saúde, custos hospitalares e tempo de hospitalização. (Ward \& Reuben 2020) Por outro lado, no que diz respeito à más indicações, podemos citar a polifarmácia, esta é fator determinante na ocorrência de interações medicamentosas indesejáveis em idosos. Isso se deve tanto à falta de conhecimento por parte desses idosos - que fazem uso de diversos fármacos sem indicação médica - quanto dos médicos que, também por falta de conhecimento em saúde do idoso, não questionam o uso dessas medicações. (Lutz et al., 2017)

Tal fato encontra-se em conformidade com um estudo realizado em Minas Gerais, Brasil, em que evidenciado a polifarmácia em $54 \%$ dos idosos estudados sendo que $67 \%$ dos idosos da amostra revelou que $67 \%$ dos medicamentos utilizados apresentavam pelo menos um risco para os idosos. (Cruz et al., 2017) Para evitar este tipo de situação é importante que o geriatra revise, a cada consulta, os medicamentos do paciente. (Rochon, 2020)

Como já mencionado, para que os idosos tenham acesso ao médico geriatra, um dos fatores influenciadores é saber a respeito da existência de uma especialidade médica para idosos. Conforme os resultados obtidos nesta pesquisa, 198 (55,5\%) entrevistados concordaram totalmente a respeito da existência de uma especialidade médica para cuidar do envelhecimento. No entanto, este número foi seguido por $24,1 \%$ dos pacientes que não estavam decididos a respeito da existência de uma especialidade para cuidar do envelhecimento e, também, por 36,1\% dos entrevistados que afirmam não estar decididos sobre quem é o médico geriatra/gerontologista.

Saber a respeito da existência da especialidade médica geriátrica e sua função é importante para que os pacientes entendam a necessidade do acompanhamento médico especializado. Tendo em vista a falta de conhecimento de boa parte do público entrevistado, 213 pacientes concordam totalmente que nunca precisaram se consultar com um médico geriatra $(59,7 \%)$ e mais 24 entrevistados afirmam não estar decididos sobre a necessidade de consulta com o médico geriatra.

Outro fator associado a consulta geriátrica é a indicação profissional ou encaminhamento deste ao médico especializado em saúde do idoso. O número de 269 pacientes que não tiveram recomendação ou encaminhamento ao médico geriatra destaca-se por se tratar de pessoas idosas. Do total de entrevistados, somente $20,2 \%$ afirmam terem sido orientadas quanto a recomendação de acompanhamento médico com o especialista.

Ademais, além das variáveis citadas, existem limitações que impedem a realização de consultas médicas especializadas por parte desses idosos. Sendo assim, ao serem questionados a respeito de fatores limitantes para se consultar com médico geriatra, 145 entrevistados $(40,6 \%)$ afirmam ter alguma limitação para se consultar com o médico geriatra, sendo que o principal fator limitante foi o acesso ao transporte (este fator foi seguido por distância ou o fato de o entrevistado morar fora da cidade de Teresina, os quais também estão intrinsicamente relacionado ao transporte), foram citadas limitações 
relacionadas ao sistema de saúde público, a necessidade e/ou disponibilidade de acompanhante, limitações relacionadas à renda e, também, limitações devido o atual estado de saúde do entrevistado. É importante mencionar que, entre os fatores limitantes também estão o não conhecimento da especialidade e a crença de não precisar de consulta com especialista de idoso ou, mesmo, de não se considerar como idoso - entre outros fatores.

Todos estes fatores obtidos por meio desta pesquisa e aqui explicitados, quando somados, refletem no total de 302 entrevistados que afirmam nunca terem se consultado com médico geriatra, mesmo possuindo idade igual ou superior a 60 anos. Após explicação a respeito de quem é o profissional geriatra e qual a sua função durante a consulta médica, 284 (79,8\%) dos entrevistados concordam totalmente que consideram importante ir ao médico geriatra.

Considerando que somente $55(15,4 \%)$ entrevistados já se consultou em algum momento da vida com este profissional, somente 30 pacientes afirmam ter regularidade em suas consultas anuais, sendo que $6(1,7 \%)$ entre os entrevistados concordam parcialmente e outros 24 (6,7\%), totalmente. A respeito da satisfação, 51 entrevistados concordam ( $0,3 \%$ parcialmente e $14 \%$ totalmente) já terem ido ao médico geriatra e ficado satisfeitos com o atendimento.

\section{Conclusão}

Dentre os objetivos desta pesquisa estava o de responder aos questionamentos "O idoso sabe que existe um profissional médico que pode cuidar de sua saúde? Caso saiba, tem acesso a ele? E qual a motivação ou a limitação para a busca por atendimento com este profissional?". Por meio dos resultados obtidos é perceptível que, somente, pouco mais da metade dos idosos entrevistados sabiam, sem dúvidas, a respeito da especialidade médica geriátrica e o papel deste profissional.

É, também, possível concluir que a falta de indicação/encaminhamento desses pacientes por profissionais de saúde é um dos fatores que limitam o acesso desses idosos ao médico geriatra, visto que somente uma minoria foi orientada a consulta com o geriatra. Para esta minoria, a recomendação de um profissional de saúde foi um dos fatores motivadores para a consulta geriátrica. Sendo assim, fica claro a necessidade da educação dos profissionais de saúde em saúde do idoso para que cada paciente de idade igual ou superior a 60 anos possa ser encaminhado ao serviço com o profissional especializado. Promovendo, assim, mais qualidade de vida na terceira idade.

Ademais, existem muitos outros fatores limitantes. Dentre eles, o destaque para o fator "transporte" chama a atenção para a necessidade de incentivo a meios adequados para este público que possui limitações de saúde específicas e relacionadas à idade. Inerente a este fator, foi citado o fato de morar fora de Teresina. Assim, é perceptível que no Piauí ainda existe grande necessidade de profissionais especializados fora dos grandes centros, sendo a geriatria mais uma entre essas especialidades medicas. Para os idosos, isso é um importante fator limitante para o acompanhamento geriátrico.

Portanto, em vista do que foi exposto nesta pesquisa científica, é perceptível a limitação tanto no conhecimento a respeito da geriatria e na motivação para estes idosos a realizarem consultas com o profissional geriatra. Desse modo, é de grande importância que as instituições de saúde promovam ações voltadas a educação em saúde do idosos, principalmente, por parte da Atenção Básica por ser porta de entrada para os demais serviços do SUS.

É importante ressaltar a carência na literatura a respeito do tema discutido neste estudo. Assim, é de grande importância que demais pesquisadores se interessem por esta temática para a melhor análise sobre a percepção de idosos a respeito da geriatria e quais fatores motivadores e/ou limitantes para o atendimento geriátrico, tanto em zonas rurais quanto urbanas, tanto em serviços públicos quanto privados. Com essas informações, pode-se realizar campanhas para que esses idosos tenham conhecimento e acesso ao médico especialista. 
Research, Society and Development, v. 11, n. 1, e10011124432, 2022

(CC BY 4.0) | ISSN 2525-3409 | DOI: http://dx.doi.org/10.33448/rsd-v11i1.24432

\section{Referências}

Brasil. (1988). Constituição da República Federativa do Brasil de 1988. Diário Oficial da República Federativa do Brasil, Brasília, 5 out. 1988.

Brasil. (1994). Lei n. 8.842, de 4 de janeiro de 1994. Dispõe sobre a política nacional do idoso, cria o Conselho Nacional do Idoso e dá outras providências. Diário Oficial da República Federativa do Brasil, Brasília, 4 jan. 1994.

Brasil. (2003). Lei n. 10.741, de 1 de outubro de 2003. Dispõe sobre o Estatuto do Idoso e outras providências. Diário Oficial da República Federativa do Brasil, Brasília, 1 out. 2003.

CRM-PI. (2021). Busca de médicos. Conselho Regional de Medicina do Estado do Piauí (CRM-PI).

Cruz, H. L., Mota, F. K. C., Araújo, L. U., Bodevan, E. C., Seixas, S. R. S., \& Santos, D. F. (2017). The utility of the records medical: factors associated with the medication errors in chronic disease. Revista latino-americana de enfermagem, 25(2967). https://doi.org/10.1590/1518-8345.2406.2967

Fonseca, S. C. (Org.), Costa, A. M. M. R., Oliveira, A. M. T., Barbosa, A. P., Côrte, B., \& Sousa, V. M. (2016). O envelhecimento ativo e seus fundamentos: documento eletrônico e impresso. https:/www.pucsp.br/sites/default/files/download/posgraduacao/programas/gerontologia/ebook__livro_o_envelhecimento_ativo_e_seus_fundamentos.pdf

Heflin, M. T. (2020) Geriatric health maintenance. Uptodate.

IBGE. (2010). Estatísticas de gênero. Instituto Brasileiro de Geografia e Estatística (IBGE).

Lutz, B. H., Miranda, V. I. A., \& Bertoldi, A. D. (2017). Potentially inappropriate medications among older adults in Pelotas, Southern Brazil. Revista de saude publica, 51(52). https://doi.org/10.1590/S1518-8787.2017051006556

Machado, K. (2019). Quem é a pessoa idosa? EPSJV/Fiocruz.

ONU. (2021). População mundial deve ter mais 2 bilhões de pessoas nos próximos 30 anos. Organização das Nações Unidas (ONU).

OPAS/OMS. (2021). Envelhecimento e saúde. Organização Pan-Americana de Saúde/ Organização Mundial da Saúde.

Pereira A. S., Shitsuka, D. M., Parreira F. J., Shitsuka R. (2018). Metodologia da pesquisa científica https://repositorio.ufsm.br/bitstream/hand le/1/15824/Lic_Computacao_Metodologia-Pesquisa-Cientifica.pdf?sequence=1.

Rochon, P. A. (2020). Drug prescribing for older adults. Uptodate.

Sá, G. G. M., Silva, F. L., Santos, A. M. R, Nolêto, J. S., Gouveia, M. T. O, \& Nogueira, L. T. (2019). Tecnologias que promovem educação em saúde para idosos comunitários: revisão integrativa. Revista latino-americana de enfermagem, 27(3186). doi: https://doi.org/10.1590/1518-8345.3171.3186

SBGG. (2021). O que é geriatria e gerontologia? Sociedade Brasileira de Geriatria e Gerontologia (SBGG).

UNFPA. (2018). Fecundidade e dinâmica da população brasileira. Fundo de População das Nações Unidas (UNFPA)

UNIFESP. (2017). Sistema Único de Saúde: histórico, diretrizes e princípios. 2017. https://www.unasus.unifesp.br/biblioteca_virtual/pab/6/ unidades_conteudos/unidade02/p_01.html

United Nations. (2019). World population prospects: the 2019 revision. Department of Economic and Social Affairs, Population Division New York.

Veras, R. P., \& Oliveira, M. (2018). Envelhecer no Brasil: a construção de um modelo de cuidado. Ciência \& Saúde Coletiva. Rio de Janeiro, 23(6) 1929. 1936. https://doi.org/10.1590/1413-81232018236.04722018

Ward, K. T., \& Reuben, D. B. (2020). Comprehensive geriatric assessment. Uptodate. 